\title{
Continuous Rotation of Achiral Nematic Liquid Crystal Droplets Driven by Heat Flux
}

\author{
Jordi Ignés-Mullol, ${ }^{1,2}$, Guilhem Poy, ${ }^{1}$ and Patrick Oswald ${ }^{1, \dagger}$ \\ ${ }^{1}$ Univ Lyon, ENS de Lyon, Univ Claude Bernard, CNRS, Laboratoire de Physique, F-69342 Lyon, France \\ ${ }^{2}$ Universitat de Barcelona, Institute of Nanoscience and Nanotechnology (IN2UB) \\ and Departament de Química Física, Martí i Franquès 1, 08028 Barcelona, Spain
}

(Received 28 March 2016; revised manuscript received 26 May 2016; published 27 July 2016)

\begin{abstract}
Suspended droplets of cholesteric (chiral nematic) liquid crystals spontaneously rotate in the presence of a heat flux due to a temperature gradient, a phenomenon known as the Lehmann effect. So far, it is not clear whether this effect is due to the chirality of the phase and the molecules or only to the chirality of the director field. Here, we report the continuous rotation in a temperature gradient of nematic droplets of a lyotropic chromonic liquid crystal featuring a twisted bipolar configuration. The achiral nature of the molecular components leads to a random handedness of the spontaneous twist, resulting in the coexistence of droplets rotating in the two senses, with speeds proportional to the temperature gradient and inversely proportional to the droplet radius. This result shows that a macroscopic twist of the director field is sufficient to induce a rotation of the droplets, and that the phase and the molecules do not need to be chiral. This suggests that one can also explain the Lehmann rotation in cholesteric liquid crystals without introducing the Leslie thermomechanical coupling-only present in chiral mesophases. An explanation based on the Akopyan and Zeldovich theory of thermomechanical effects in nematics is proposed and discussed.
\end{abstract}

DOI: 10.1103/PhysRevLett.117.057801

Molecular rotors and motors [1], such as the proteins that drive active self-organization in the cell cytoskeleton, are nanoscale entities that transform energy from a surrounding source into directed motion. Among the incipient manmade attempts at replicating such behavior, a remarkable example is endowed by chiral-nematic, or cholesteric, liquid crystals (CLC), whose director field can be set into rotation by gradients of scalar fields [2]. Recent experimental realizations include chemomechanical coupling both in Langmuir monolayers $[3,4]$ and in thin films of chiral molecules [5], or electromechanical coupling in different bulk and disperse systems [6-8], although the interpretation of the latter type of experiments often remains unclear [9-13]. Perhaps the best known among these intriguing nonequilibrium thermodynamic phenomena is the thermomechanical coupling, known as the Lehmann effect, described in 1900 by Lehmann [14], who reported the continuous rotation of the internal texture of CLC droplets in equilibrium with the isotropic phase, when they were subjected to a temperature gradient. In spite of the apparent simplicity of these observations, they remained experimentally elusive until recently [15-18].

From a theoretical perspective, Leslie's generalization of nematohydrodynamics to CLC $[19,20]$ demonstrated that the absence of mirror and inversion symmetries enable the induction of a torque on the CLC director by means of a thermal gradient. The assumption that this torque was responsible for the Lehmann effect became a paradigm in the literature. Recent results, however, show that the Leslie coupling cannot be entirely responsible for the
Lehmann effect, in particular because the first one is tied to the macroscopic chirality of the phase, independently of the macroscopic twist $q$ of the director field [21-24], whereas the second one crucially depends on $q$. This was evidenced by the fact that the Leslie coupling is still observable at the compensation temperature of a cholesteric phase where $q=0$ [21-23], whereas the Lehmann rotation disappears at this point $[25,26]$. In addition, the Leslie torque and the rotation vector of the droplets are sometimes of opposite sign [24]. As a consequence, these two effects must be clearly distinguished even if both seem to come from the chirality of the phase and the molecules.

An important question thus arises: can we observe the Lehmann and(or) Leslie effects in a phase-chiral or achiral — of a material made of achiral molecules? A part of the answer has been recently suggested by Brand et al. [27], who show theoretically that one might expect thermomechanical effects similar to the Leslie coupling of CLC in chiral phases of achiral molecules, such as those formed by banana-shaped molecules [28]. But this does not mean that a Lehmann effect can exist in these phases if the Leslie paradigm is wrong.

Here, we show that it is possible to observe the Lehmann rotation in a usual nematic LC, where both the molecules and the phase — of the $D_{\infty h}$ symmetry-are achiral. In the absence of molecular-level chiral induction, the required twist in the director field must be provided by topological constraints. For example, this can be the case inside tangentially anchored bipolar nematic droplets provided that the twist constant, $K_{2}$, is much smaller than the splay 
and bend constants, $K_{1}$ and $K_{3}$ [29]. Experimentally, this condition is fulfilled in the nematic phase of the lyotropic LC made with aqueous suspensions of the food dye Sunset Yellow FCF (SSY) [30], where twisted bipolar droplets nucleate in the coexistence region with the isotropic liquid. We recall that SSY is the commercial name of the disodium salt of 6-hydroxy-5-[(4-sulfophenyl)azo]-2-naphtalenesulfonic acid. The compound was purchased from Sigma-Aldrich, and purified by successive recrystallizations of saturated water solutions using ice-cold absolute ethanol. The wet precipitate was dried at $120^{\circ} \mathrm{C}$ under vacuum during a day. Aqueous solutions of SSY (molar mass 452.37) were prepared with concentrations typically ranging between 0.88 and $1.0 \mathrm{~mol} / \mathrm{kg}$, which results in the nematic-isotropic coexistence above room temperature. Because of their flat central core of polyaromatic rings, SSY molecules tend to form aggregates by face-to-face stacking in water. These elongated aggregates may self-assemble into ordered mesophases, constituting the so-called lyotropic chromonic liquid crystals (LCLC) [31].

In our experiments, the LCLC was contained in a rectangular cell of gap $110 \mu \mathrm{m}$, whose glass plates were spin coated with polyvinyl alcohol (PVA) and annealed at $120^{\circ} \mathrm{C}$ for $1 \mathrm{~h}$ in order to favor wetting of the walls by the isotropic phase. The cell was placed between the two transparent ovens of the experimental setup described in Ref. [15]. A red bandpass filter was placed in front of the microscope light source to optimize optical contrast and to minimize local heating of the sample. Two thin layers of glycerol ensured a good thermal contact between the cell and the ovens. The temperature gradient, perpendicular to the cell walls, is given by $G=(\Delta T / 4 e)\left(\kappa_{g} / \kappa_{\mathrm{LC}}\right)$, where $e=1 \mathrm{~mm}$ is the thickness of the glass plates, $\Delta T=T_{u}-T_{b}$ is the temperature difference between the upper and bottom ovens and $\kappa_{g}$ and $\kappa_{\mathrm{LC}}$ are the thermal conductivities of the glass and the LC, respectively. By taking for $\kappa_{\mathrm{LC}}$ the conductivity of water, we obtain $\left(\kappa_{g} / \kappa_{\mathrm{LC}}\right) \sim 1 / 0.6$, which gives $G(\mathrm{~K} / \mathrm{m}) \sim 420 \Delta T(\mathrm{~K})$.

In order to observe the Lehmann rotation, the cell is heated in the nematic-isotropic coexistence region. In the studied LCLC, the coexistence range is relatively large, of the order of $10-12{ }^{\circ} \mathrm{C}$. Most of our observations are made at a temperature that falls roughly in the middle of the coexistence range. Nematic droplets are at rest when they nucleate under uniform temperature conditions, and start to rotate when a temperature gradient is imposed, revealing the Lehmann effect. Contrary to what is observed in CLC, we observe coexistence of droplets rotating in opposite senses. This is visible in Fig. 1, and in video S1 (scale bar $20 \mu \mathrm{m}$ ) of the Supplemental Material [32], where two neighboring droplets rotate with opposite handedness. We argue that the rotation is due to the spontaneous twist of the droplet director field, which can be positive or negative with the same likelihood, since the nematic phase is achiral.

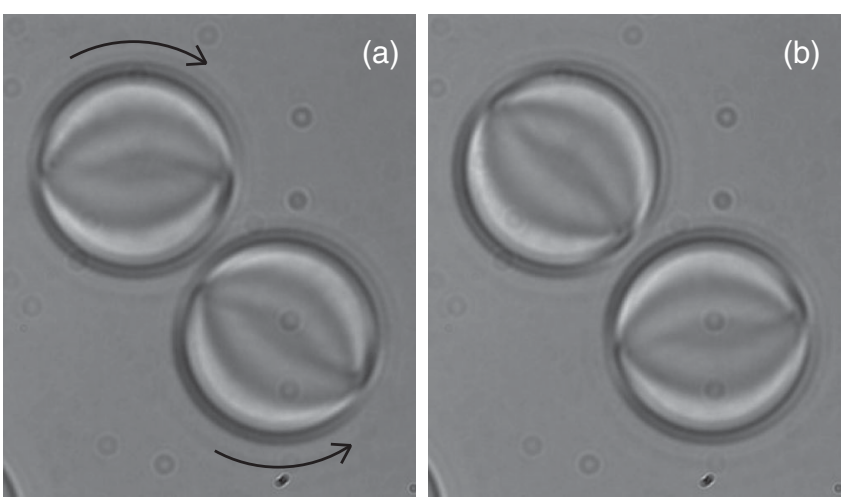

FIG. 1. Optical micrograph of two twisted bipolar droplets of radius $25 \mu \mathrm{m}$ rotating in opposite directions. (a) $t=0$; (b) $t=10 \mathrm{~s}$. $T_{\mathrm{NI}}=45^{\circ} \mathrm{C}$ and $\Delta T=20^{\circ} \mathrm{C}$. Images are taken using unpolarized red light illumination. The axis of the droplets lays on the image plane.

Combined with the results reported for CLC, our experiments prove that the Lehmann rotation is due to the chirality of the director field and not to the chirality of the phase, thus confirming that the Leslie mechanism, which is only possible in a chiral phase, cannot explain alone the Lehmann rotation observed in CLC.

To go further in the analysis of the phenomenon, we have explored the influence of the temperature gradient, the droplet radius, and the elastic constants (through their temperature dependence) on the period of rotation, $\Theta$, of the nematic droplets. Figure 2 shows data for a sample with $T_{\mathrm{NI}} \approx 45^{\circ} \mathrm{C}$ (SSY concentration $\approx 0.95 \mathrm{~mol} / \mathrm{kg}$ ) in different temperature gradients. Here, $T_{\mathrm{NI}}$ corresponds to the center of the nematic-isotropic coexistence region. Temperature difference, $\Delta T$, ranges from $2.5^{\circ} \mathrm{C}$ until $40^{\circ} \mathrm{C}$. For a given $\Delta T$, the steady-state droplet size is increased (decreased) by slightly decreasing (increasing) the mean temperature around $45^{\circ} \mathrm{C}$, while maintaining a constant $\Delta T$. Each data set is fitted with a straight line with zero intercept. At least half a turn is analyzed for each rotating droplet, and only droplets with a well-oriented bipolar configuration-with the dipole axis perpendicular to $\vec{G}$-are considered. We observe that higher $G$ favors droplets oriented with their axis either parallel or perpendicular to $\vec{G}$. At lower $G$, droplets with intermediate orientations are abundant but we do not analyze them because their tilt angle usually changes during the recording. Note that the droplets with their axis parallel to $\vec{G}$ do not rotate, which is expected since the director field is invariant under rotation about $\vec{G}$. We also note that large droplets are unstable with respect to coalescence in high $G$. This explains why the range of measured radii decreases when $G$ increases. Figure 2(a) shows that $\Theta$ varies linearly with $R$ for each $\Delta T$, and increases when $G$ decreases. Figure 2(b) shows that it is possible to collapse all these data on the same master curve by plotting $\Theta \Delta T$ as a 


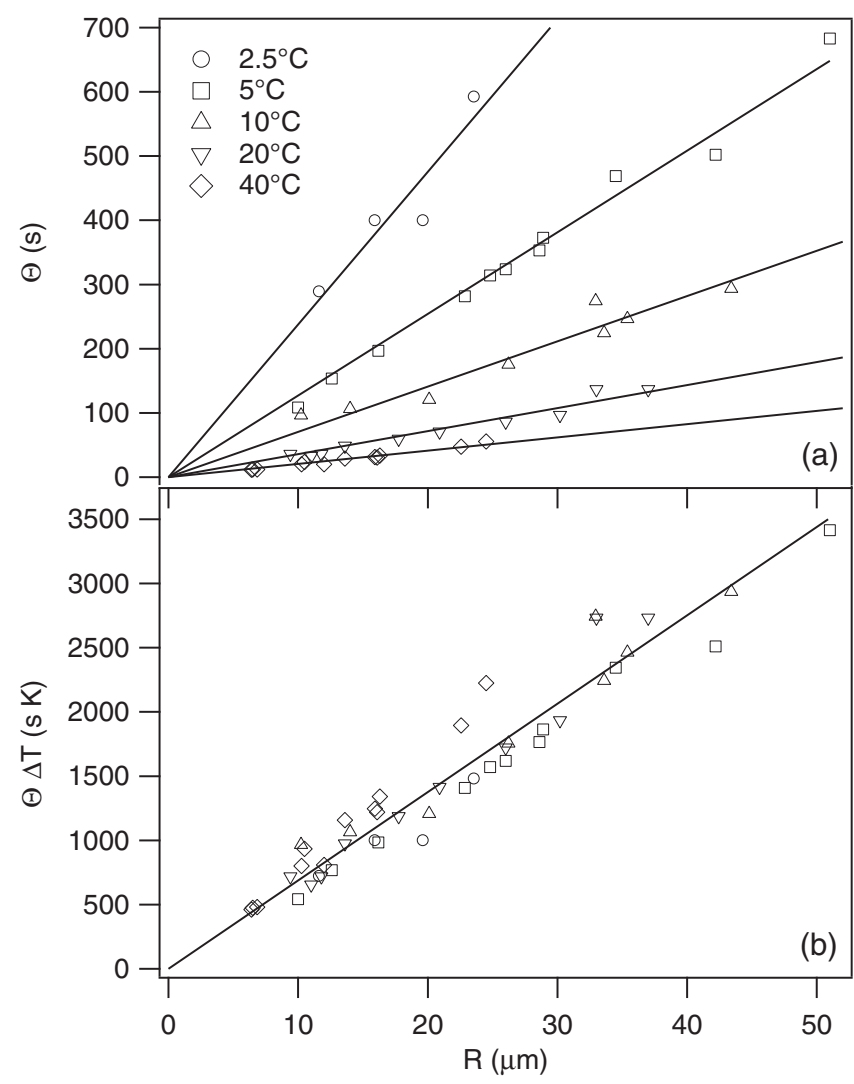

FIG. 2. (a) Period of rotation, $\Theta$, as a function of droplet radius, $R$, for different temperature difference $\Delta T$, and their fit to a straight line passing through the origin. (b) Product $\Theta \Delta T$ as a function of $R$. $T_{\mathrm{NI}}=45^{\circ} \mathrm{C}$.

function of $R$. This proves that $\Theta$ is inversely proportional to $G$ or, equivalently, that the rotation velocity is proportional to $G$. Finally, the period $\Theta$ for mixtures with different SSY concentrations, and so different coexistence temperatures and elastic constants, is plotted in Fig. 3. Each data

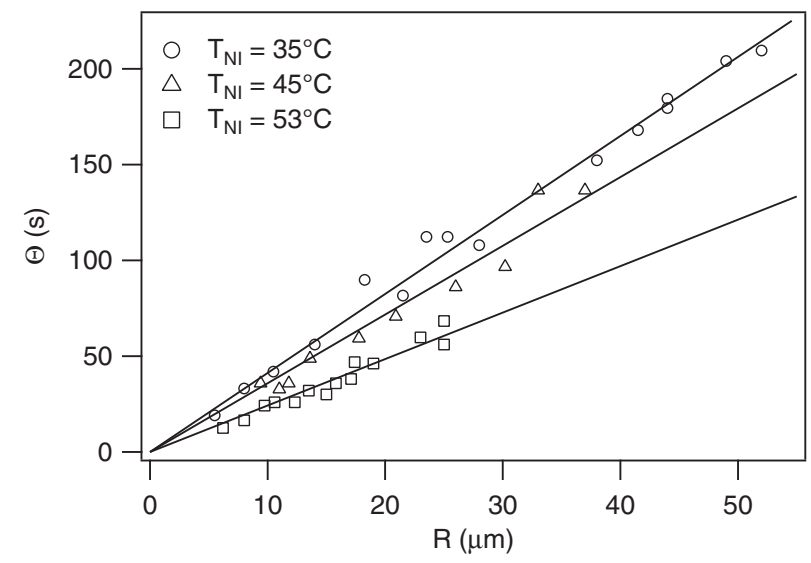

FIG. 3. Period of rotation as a function of the radius at $\Delta T=$ $20^{\circ} \mathrm{C}$ and different concentrations of SSY corresponding to different $T_{\mathrm{NI}}$. Circle: $T_{\mathrm{NI}}=35^{\circ} \mathrm{C}$, SSY conc. $0.88 \mathrm{~mol} / \mathrm{kg}$; Triangle: $T_{\mathrm{NI}}=45^{\circ} \mathrm{C}, \quad \mathrm{SSY}$ conc. $0.95 \mathrm{~mol} / \mathrm{kg}$; Square: $T_{\mathrm{NI}}=53^{\circ} \mathrm{C}$, SSY conc. $1.0 \mathrm{~mol} / \mathrm{kg}$. set is taken at the same temperature gradient $\left(\Delta T=20^{\circ} \mathrm{C}\right)$. This graph shows that the droplets slow down (the period of rotation increases) when the SSY concentration (or equivalently, the transition temperature $T_{\mathrm{NI}}$ ) decreases.

In order to explain these data, we adapt the model of Ref. [15], originally developed for CLC, to the twisted bipolar nematic droplets. In CLC, the driving torque for the rotation of the director is the Leslie thermomechanical torque given by $\vec{\Gamma}_{\text {Leslie }}=\nu \vec{n} \times(\vec{n} \times \vec{G})$, where $\vec{n}$ is the director, and $\nu$ is the so-called Leslie thermomechanical coefficient. In the nematic case, this torque vanishes for symmetry reasons $(\nu=0)$. Nevertheless, Akopyan and Zeldovich have shown that, in deformed nematics, the director can still experience a thermomechanical torque of general expression [33]:

$$
\vec{\Gamma}_{\mathrm{TM}}=\vec{n} \times \vec{f}_{\mathrm{TM}}
$$

where the thermomechanical force is given by

$$
\begin{aligned}
\vec{f}_{\mathrm{TM}}= & \left(-\xi_{1}+\frac{\xi_{3}}{2}\right)(\vec{\nabla} \cdot \vec{n}) \vec{G}+\xi_{2}(\vec{n} \cdot \vec{\nabla} \times \vec{n})(\vec{n} \times \vec{G}) \\
& +\frac{1}{2}\left(\xi_{4}-\xi_{3}\right)(\vec{n} \times(\vec{\nabla} \times \vec{n}))(\vec{n} \cdot \vec{G})-\xi_{3} \underline{\underline{m}} \vec{G} .
\end{aligned}
$$

In this expression, $\underline{m}$ is the symmetric tensor of components $m_{i j}=\left(n_{i, j}+n_{j, i}\right) / 2$, and the $\xi_{i}$ 's $(i=1 \ldots 4)$ are phenomenological constants that account for the different modes of director distortion that may give rise to thermomechanical torques. We emphasize that a more exact derivation of expression (2) has been given by Pleiner and Brand in Ref. [34], p. 44. In this work, the thermomechanical coefficients are denoted by $\Pi_{i}(i=1 \ldots 4)$ with the correspondence $\xi_{1}=\gamma_{1}\left(\Pi_{1}+\Pi_{2} / 2+\Pi_{3} / 2\right), \xi_{2}=\gamma_{1}\left(\Pi_{2}-\Pi_{3}\right) / 2, \xi_{3}=$ $\gamma_{1}\left(\Pi_{2}+\Pi_{3}\right)$, and $\xi_{4}=2 \gamma_{1} \Pi_{4}$. By assuming that there is no flow-as in banded and oriented droplets of CLC [35] - we obtain that the texture rotates at an angular velocity $\omega$ given by

$$
\gamma_{1} \omega=\frac{\int_{V} \vec{f}_{\mathrm{TM}} \cdot \vec{\delta} d^{3} \vec{r}}{\int_{V} \vec{\delta}^{2} d^{3} \vec{r}},
$$

where $V$ is the volume of the droplet and $\vec{\delta}=\vec{e}_{z} \times \vec{n}-$ $\partial \vec{n} / \partial \vartheta$. Here, $\vartheta$ is the polar angle in cylindrical coordinates centered on the droplet $(r, \vartheta, z)$, and the unit vector $\vec{e}_{z}$ is parallel to the $z$ axis, which is perpendicular to the glass plates.

Expressions (2) and (3) may be used to calculate the rotation velocity of the twisted bipolar droplets for a given configuration of the director field inside the droplets. In order to simplify the calculations, we suppose that the planar anchoring at the droplet surface is strong and use an ansatz for the director field that combines a pure bipolar configuration, $\vec{n}_{b}$, and a pure concentric configuration, $\vec{n}_{c}$, namely, $\vec{n}_{\mathrm{tb}}=\vec{n}_{b} \cos (\alpha)-\vec{n}_{c} \sin (\alpha)$, with $\alpha(\rho)=\alpha_{0} \rho / \rho_{0}$ 
$[36,37]$. Here, $\rho$ is the radius in the cylindrical coordinates local to the droplet $(\rho, \varphi, \zeta)$, with the $\zeta$ axis taken along the bipole, and $\rho_{0}=\sqrt{R^{2}-\zeta^{2}}(-R \leq \zeta \leq R)$. The angle $\alpha_{0}$ fixes the twist inside the droplet (inset in Fig. 4). This angle can be estimated by minimizing the Frank elastic energy of the droplet [37], and it is found to only depend on the elastic anisotropy of the nematic phase, independently of the droplet radius. With typical values of the elastic constants of the LCLC given in Ref. [30], we estimated $\alpha_{0} \sim 70^{\circ}$. We also measured $\alpha_{0}$ experimentally by analyzing the transmittance through the center of droplets, and considering a twisted planar nematic configuration in the Mauguin regime, in which the polarization of light follows the director [37]. Using values for SSY birefringence reported in the literature [38], our analysis yields $\alpha_{0}=$ $100^{\circ} \pm 3^{\circ}$ in the range of concentrations used here, which is of the same order as the previous estimate. Introducing the above approximation for the director field $\vec{n}_{t b}$ in Eqs. (1)-(3) yields

$$
-\frac{\gamma_{1} R \omega}{G}=\frac{\sum_{i} \bar{\xi}_{i} J_{i}\left(\alpha_{0}\right)}{J_{d}\left(\alpha_{0}\right)},
$$

with $\bar{\xi}_{1}=-\xi_{1}-\left(\xi_{3} / 2\right), \bar{\xi}_{2}=\xi_{2}-\left(\xi_{3} / 2\right), \bar{\xi}_{3}=-\left(\xi_{4} / 2\right)$, and

$$
\begin{gathered}
J_{1}=\int_{V_{1}}\left(\vec{\nabla} \cdot \vec{n}_{t b}\right)\left(\vec{e}_{z} \cdot \frac{\partial \vec{n}_{t b}}{\partial \vartheta}\right) d^{3} \vec{r}^{\prime}, \\
J_{2}=\int_{V_{1}}\left(\vec{n}_{t b} \cdot \vec{\nabla} \times \vec{n}_{t b}\right)\left[\left(\vec{e}_{z} \times \vec{n}_{t b}\right) \cdot \vec{\delta}\right] d^{3} \vec{r}^{\prime},
\end{gathered}
$$

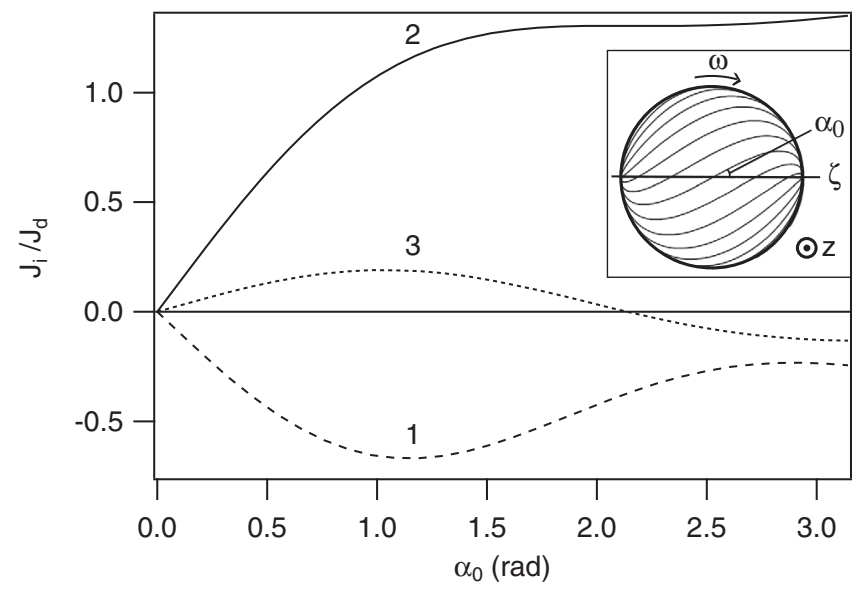

FIG. 4. Ratios of the splay $(i=1)$, twist $(i=2)$, and bend $(i=3)$ integrals $J_{i}$ over the dissipation integral $J_{d}$ as a function of the twist angle $\alpha_{0}$. The value of $i$ is indicated beside each curve. Inset: angle $\alpha_{0}$ and director surface field lines in a twisted bipolar droplet. The $\zeta$ axis is the bipole axis and the $z$ axis is perpendicular to the glass plates and parallel to the temperature gradient.

$$
\begin{gathered}
J_{3}=\int_{V_{1}}\left(\vec{n}_{t b} \cdot \vec{e}_{z}\right)\left[\vec{n} \times\left(\vec{\nabla} \times \vec{n}_{t b}\right) \cdot \vec{\delta}\right] d^{3} \vec{r}^{\prime}, \\
J_{d}=\int_{V_{1}} \vec{\delta}^{2} d^{3} \vec{r}^{\prime} .
\end{gathered}
$$

Note that the integrals $J_{i}\left(J_{d}\right)$, which are odd (even) functions of $\alpha_{0}$, are made dimensionless by using the variables $x_{i}^{\prime} \equiv x_{i} / R$ and are calculated over a spherical volume $V_{1}$ of radius unity. The calculated ratios $J_{i} / J_{d}$, evaluated using Mathematica, are shown in Fig. 4 as a function of the surface twist angle $\alpha_{0}$. They all vanish when $\alpha_{0}=0$, which means that $\omega=0$ when the droplet is not twisted. This is expected because, in that case, the droplet is superposable to its image in a mirror containing the temperature gradient. But, since the droplet and its mirror image must have opposite velocities, this results in $\omega=-\omega=0$. Equation (4) shows that, in agreement with experiments, the rotation velocity $\omega$ is proportional to $G$ and inversely proportional to $R$. It must be emphasized that the dependence in $R$ arises from purely dimensional reasons in this model. Indeed, the only dimensionless quantity that can be formed with the variables $R, G, \omega$, and the material constants $\gamma_{1}$ and $\bar{\xi}$ (with dimensions $\mathrm{N} \mathrm{m}^{-2} \mathrm{~s}$ and $\mathrm{N} \mathrm{K}^{-1}$, respectively) is $\gamma_{1} R \omega /(\bar{\xi} G)$. This result holds if $R$ is the only length scale in the problem, which supposes that the anchoring extrapolation length is much smaller than $R$ (strong anchoring). From our experimental data shown in Fig. 2(b), we can estimate a value for the ratio $\bar{\xi} / \gamma_{1}$, by assuming that all the $\bar{\xi}_{i}$ are equal, and by taking $\alpha_{0} \sim 100^{\circ}$ we get $\left(\bar{\xi} / \gamma_{1}\right) \approx 2.5 \times 10^{-10} \mathrm{~m}^{2} \mathrm{~s}^{-1} \mathrm{~K}^{-1}$. We measured the rotational viscosity with a rotating magnetic field according to the method described in Ref. [39] and by taking the value of the magnetic anisotropy given in Ref. [30] we get $\gamma_{1} \approx 0.32 \mathrm{~Pa}$. This gives $\bar{\xi} \approx 8 \times 10^{-11} \mathrm{~N} \mathrm{~K}^{-1}$. We obtained the sign of $\bar{\xi}$ by doping the LCLC with a small amount of the chiral dopant brucine sulphate. We verified, by measuring the twist angle in cells with homogeneous planar unidirectional (rubbed glass)-planar sliding (PVA coating) anchoring, that this substance promotes a right-handed twist in SSY. In the presence of this dopant, most of the droplets rotate in the same direction, with $\omega$ and $G$ of opposite signs, which leads to $\bar{\xi}>0$. We emphasize that our value of $\bar{\xi}$ is 50 times larger in absolute value than that measured experimentally in hybrid layers of nematic LC by Lavrentovich and Nastishin [40] and by Akopyan et al. [41]. This leads us to think that this model is, at most, qualitatively correct, and that another mechanism is involved as in the classical Lehmann effect. Only independent and more precise measurements of the coefficients $\bar{\xi}_{i}$, for instance in the geometry proposed by Poursamad in Ref. [42], will enable us to settle this issue.

In conclusion, the Lehmann effect also exists in usual nematic LC. In the experiments reported here, the rotation 
is due to a macroscopic chiral symmetry breaking of the director field inside droplets with twisted bipolar director field. This configuration arises in SSY LCLC thanks to their giant elastic anisotropy. The main difference with respect to CLC is that here droplets rotating in both directions coexist in the same sample since their spontaneous twist develops indifferently to the right or to the left. This observation shows that the phase and the molecules do not need to be chiral to observe a Lehmann rotation. This is different from the Leslie thermomechanical effect, which can only be observed in chiral mesophases. In the future, it would be interesting to measure more precisely the thermomechanical coefficients $\xi_{i}$ introduced by Akopyan and Zel'dovich in usual nematics. Another question concerns the existence of similar terms in CLC. In principle, they should also exist in CLC but it is not clear how to generalize Eq. (2) to this phase. In particular, it is not clear whether the thermomechanical force $\vec{f}_{\mathrm{TM}}$ must be "regularized" in order that it vanishes in a nondeformed cholesteric phase, the term in $\xi_{3}$ becoming problematic. Once this theoretical problem is resolved, it would be interesting to check the role of these terms in the classical Lehmann effect. Nevertheless, we think that the thermomechanical terms are not necessary to explain the Lehmann effect both in nematic and cholesteric LCs.

We thank A. Dequidt and E. Kats for fruitful discussions and B. Martínez-Prat for assistance in the characterization of SSY samples. J. I-M. acknowledges support by the CNRS for hosting his visit to the Physics laboratory of the ENS of Lyon. This work was partially supported by project MINECO-FIS2013-41144P (J. I-M.).

*jignes@ub.edu patrick.oswald@ens-lyon.fr

[1] J. Milch and E. C. H. Sykes, ACS Nano 3, 1042 (2009).

[2] P.-G. de Gennes, The Physics of Liquid Crystals (Oxford University Press, New York, 1974).

[3] Y. Tabe and H. Yokoyama, Nat. Mater. 2, 806 (2003).

[4] P. Milczarczyk-Piwowarczyk, A. Żywociński, K. Noworyta, and R. Holyst, Langmuir 24, 12354 (2008).

[5] K. Seki, K. Ueda, Y.-i. Okumura, and Y. Tabe, J. Phys. Condens. Matter 23, 284114 (2011).

[6] L. Gil and S. Thiberge, J. Phys. II (France) 7, 1499 (1997).

[7] N. V. Madhusudana and R. Pratibha, Mol. Cryst. Liq. Cryst. 5, 43 (1987).

[8] H. P. Padmini and N. V. Madhusudana, Liq. Cryst. 14, 497 (1993).

[9] L. Gil and J. M. Gilli, Phys. Rev. Lett. 80, 5742 (1998).

[10] J. Baudry, S. Pirkl, and P. Oswald, Phys. Rev. E 60, 2990 (1999).

[11] O. S. Tarasov, A. P. Krekhov, and L. Kramer, Phys. Rev. E 68, 031708 (2003).
[12] O. S. Tarasov, Structural Transition and Dynamics of Liquid Crystals under Flows and Electric Fields, Ph.D. thesis, University of Bayreuth, 2003.

[13] A. Dequidt and P. Oswald, Eur. Phys. J. E 24, 157 (2007).

[14] O. Lehmann, Ann. Phys. (Berlin) 307, 649 (1900).

[15] P. Oswald and A. Dequidt, Phys. Rev. Lett. 100, 217802 (2008).

[16] P. Oswald, Eur. Phys. J. E 28, 377 (2009).

[17] J. Yoshioka, F. Ito, Y. Suzuki, H. Takahashi, H. Takizawa, and Y. Tabe, Soft Matter 10, 5869 (2014).

[18] T. Yamamoto, M. Kuroda, and M. Sano, Europhys. Lett. 109, 46001 (2015).

[19] F. M. Leslie, Arch. Ration. Mech. Anal. 28, 265 (1968).

[20] F. M. Leslie, Symp. Faraday Soc. 5, 33 (1971).

[21] N. Éber and I. Jánossy, Mol. Cryst. Liq. Cryst. Lett. 72, 233 (1982).

[22] A. Dequidt and P. Oswald, Europhys. Lett. 80, 26001 (2007).

[23] P. Oswald and A. Dequidt, Europhys. Lett. 83, 16005 (2008).

[24] P. Oswald, Europhys. Lett. 108, 36001 (2014); 108, 59901 (2014).

[25] P. Oswald, L. Jorgensen, and A. Żywociński, Liq. Cryst. 38, 601 (2011).

[26] P. Oswald, Eur. Phys. J. E 35, 10 (2012).

[27] H. Brand, H. Pleiner, and D. Svenšek, Phys. Rev. E 88, 024501 (2013).

[28] G. Pelzl, S. Diele, and W. Weissflog, Adv. Mater. 11, 707 (1999).

[29] R. D. Williams, J. Phys. A: Math. Gen. 19, 3211 (1986).

[30] S. Zhou and co workers, Phys. Rev. Lett. 109, 037801 (2012).

[31] H.-S. Park and O. Lavrentovich, Lyotropic chromonic liquid crystals: Emerging applications, in Liquid Crystals Beyond Displays: Chemistry, Physics, and Applications, edited by Q. Li (John Wiley \& Sons, New York, 2012), Chap. 14.

[32] See Supplemental Material at http://link.aps.org/ supplemental/10.1103/PhysRevLett.117.057801 showing two neighboring droplets rotating in opposite directions.

[33] R. S. Akopyan and B. Y. Zel'dovich, Sov. Phys. JETP 60, 953 (1984).

[34] H. Pleiner and H. R. Brand, in Pattern Formation in Liquid Crystals (Springer, New York, 1996), p. 15.

[35] G. Poy and P. Oswald, Soft Matter 12, 2604 (2016).

[36] F. Xu and P. Crooker, Phys. Rev. E 56, 6853 (1997).

[37] J. Jeong, Z.S. Davidson, P. J. Collings, T. C. Lubensky, and A. G. Yodh, Proc. Natl. Acad. Sci. U.S.A. 111, 1742 (2014).

[38] V. R. Horowitz, L. A. Janowitz, A. L. Modic, P. A. Heiney, and P. J. Collings, Phys. Rev. E 72, (2005).

[39] P. Oswald, Europhys. Lett. 100, 26001 (2012).

[40] O. D. Lavrentovich and Y. A. Nastishin, Ukr. Fiz. Zh. 32, 710 (1987).

[41] R. S. Akopyan, R. B. Alaverdian, E. A. Santrosian, and Y. S. Chilingarian, J. Appl. Phys. 90, 3371 (2001).

[42] J. B. Poursamad, Journal of Contemporary Physics;Izvestiya Akademii Nauk Armenii, Fizika 44, 14 (2009). 